\title{
The Human Hand and Foot in Evolution and Art: The Effects of Wearing Footwear
}

\author{
Clifford Qualls ${ }^{1}$, Peter Evans ${ }^{2}$, Antonio Perciaccante ${ }^{3}$, Raffaella Bianucci ${ }^{4-6}$, Donatella Lippi ${ }^{7}$ and Otto Appenzeller ${ }^{8^{*}}$ \\ ${ }^{1}$ Health Sciences Center, University of New Mexico, Albuquerque NM, USA \\ 226 Windsor Cres Surrey Hills, VIC3127, Melbourne, Australia \\ ${ }^{3}$ Department of Medicine, San Giovanni di Dio Hospital, Gorizia, Italy \\ ${ }^{4}$ Warwick Medical School, Microbiology and Infection Division, University of Warwick, United Kingdom \\ ${ }^{5}$ Legal Medicine Section, Department of Public Health and Paediatric Sciences, University of Turin, Italy \\ ${ }^{6}$ Laboratoire d'Anthropologie bio-culturelle, Droit, Etique\& Sante (Ades), Faculte de Medecine de Marseille, France \\ ${ }^{7}$ Department of Experimental and Clinical Medicine, University of Florence, Italy \\ ${ }^{8}$ New Mexico Health Enhancement and Marathon Clinics (NMHEMC) Research foundation, Albuquerque, NM 87122, USA
}

"Corresponding author: Otto Appenzeller, New Mexico Health Enhancement and Marathon Clinics (NMHEMC) Research foundation, Albuquerque, NM 87122, USA, Tel: 5517-981666732; E-mail: m.zotarelli@gmail.com

Received date: 12 March 2018; Accepted date: 27 March 2018; Published date: 04 April 2018

Copyright: ( 2018 Qualls C, et al. This is an open-access article distributed under the terms of the creative commons attribution license, which permits unrestricted use, distribution and reproduction in any medium, provided the original author and source are credited.

\begin{abstract}
The structures of the human hands and feet are shaped by evolution and its effects on the brain, skeleton and other structures, and on behavior.

We used measurements obtained of hands and feet from living humans in Europe, the Americas (South and North) and Australia and images of hands and feet in cave art, paintings, and photographs obtained from the Web including some from Africa. We used the ratios of the third finger/width of hand and second toe/width of foot. We hypothesized that hand ratios would not have changed over millennia whereas, because of the use of footwear and mechanical locomotion, the ratios obtained from feet could have changed significantly. Here we report that statistical analyses and modeling confirmed our initial hypothesis.
\end{abstract}

Keywords: Hands; Feet; Spine; Footwear

\section{Introduction}

Obligatory bipedal posture and locomotion found in humans has resulted in evolutionary changes affecting the structure of the hands, of the feet and of other skeletal parts such as the spine and freed the hands for participation in artistic endeavors [1]. Comparisons of hands in primates has recently led to the conclusion that the human hand has retained many ancient modifications whereas the feet have changed significantly in modern times [2]. The foot has, throughout evolution, aided primarily locomotion but has not participated significantly in artistic and symbolic endeavors such as gestures.

Multiple regions of the brain are involved in art and career artists largely retain artistic endeavors after brain damage such as stroke and injuries. Thus redundancy of cerebral connectivity likely ensures continued function after localized cerebral damage [3]. We hypothesized that measures of the ratios, width of hand/length of 3rd finger, and width of foot/length of 2nd toe in living humans compared to similar ratios depicted in ancient cave art, photographs and paintings obtained from the World Wide Web and from art books might give insight into changes in these ratios wrought by brain function and by artistic endeavors.

We used measurements obtained from living humans in Europe, the Americas (South and North) and Australia and images of hands and feet in cave art, paintings, and photographs obtained from the Web including some from Africa.

We hypothesized that hand ratios would not have changed over time whereas, because of the use of footwear and mechanical locomotion, the ratios obtained from feet could have changed significantly. Here we report that statistical analyses and modeling confirmed our initial hypothesis.

\section{Methods}

We obtained IRB approval from the New Mexico Health Enhancement and Marathon Clinics Research Foundation (NMHEMC Research Foundation) for the measurements of hands and feet in modern subjects using calipers. Informed written consent was obtained from living subjects.

We based our analyses on 221 data points available from measurements of dominant hands and feet in modern people from Europe, the Americas, Australia and Africa. We supplemented our measurements of hands and feet in various media (photos, paintings, cave stencils, chiseled images and images of bas-reliefs) gleaned from the World Wide Web (www.com) of ancient images and modern images (Table 1).

In living people (modern), our gold standard, we used calipers (General $^{\circ}$ stainless steel digital Fractional Caliper; precision \pm 0.02 $\mathrm{mm}$; resolution $0.01 \mathrm{~mm}$ ). Images were measured using Adobe Acrobat measuring tool precision $\pm 0.01 \mathrm{~mm}$. We determined the 
dominant hand by asking subjects "which hand they used to handle a spoon" and which "foot they kicked a ball with" [3]. We also divided this group arbitrarily into young to mature (18-59) and old $(\geq 60)$ and into males and females and into manual workers and non-manual workers. We recruited our subjects from the Americas (North and South) Europe and Australia (Table 1).

\begin{tabular}{|l|l|l|l|l|l|l|}
\hline & $\begin{array}{c}\text { Modern M } \\
\text { Stencils }\end{array}$ & Paintings & Photos & Chiseled & Rocks & Total \\
\hline Africa & & & & 6 & & 6 \\
\hline Americas & 70 & 11 & & 3 & 6 & 89 \\
\hline Australia & 20 & 35 & 13 & 1 & 5 & 75 \\
\hline Europe & 16 & 24 & 11 & & & 51 \\
\hline
\end{tabular}

Table 1: Measures obtained from hands and feet in various continents and in media such as cave art paintings photos, chiseled/rocks (total 221).

\section{Statistical Methods}

Frequency tables were used to count and Analysis of Variance (ANOVA) was used for the analyses of continuous measures (hand and foot ratios) and to obtain P-values. The factors in these analyses included four continents, six media types, hand and foot dominance, two age groups in modern persons, and period as a continuous covariable. $P$-values $\leq 0.05$ were considered significant.

\section{Results}

\section{Hands}

The ratios in manual workers were larger $(\mathrm{P}=0.45)$ in modern subjects. There were no statistically significant differences in the ratios of the hands between right and left dominant hands $(\mathrm{P}=0.9)$. Old $(>60$ years) and young to mature (18-59 years) had no differences in hand ratios $(\mathrm{P}=09)$. There were no differences in old versus young manual workers $(\mathrm{P}=0.8)$. In measurements obtained from the worldwide web the status of the manual versus non manual, the age and hand dominance could not be assessed.

\section{Period}

We assessed hand ratios at various time periods spanning from the present to 40,000 years ago (Figure 1).

There were no statistical differences between the present and distant past in the slope.

\section{Feet}

We did similar analyses for foot ratios. The measurements of foot ratios in living people (modern) showed significant differences between manual and non manual workers in Europe $(\mathrm{P}>0.001)$ but not in other locations.

There was a difference between young to mature and old subjects $(\mathrm{P}=.045)$. We had no information about foot dominance in Web derived images. We then analyzed the relationship of foot ratios to period. The slope was significant $(\mathrm{P}<0.0001)$ (Figure 2).

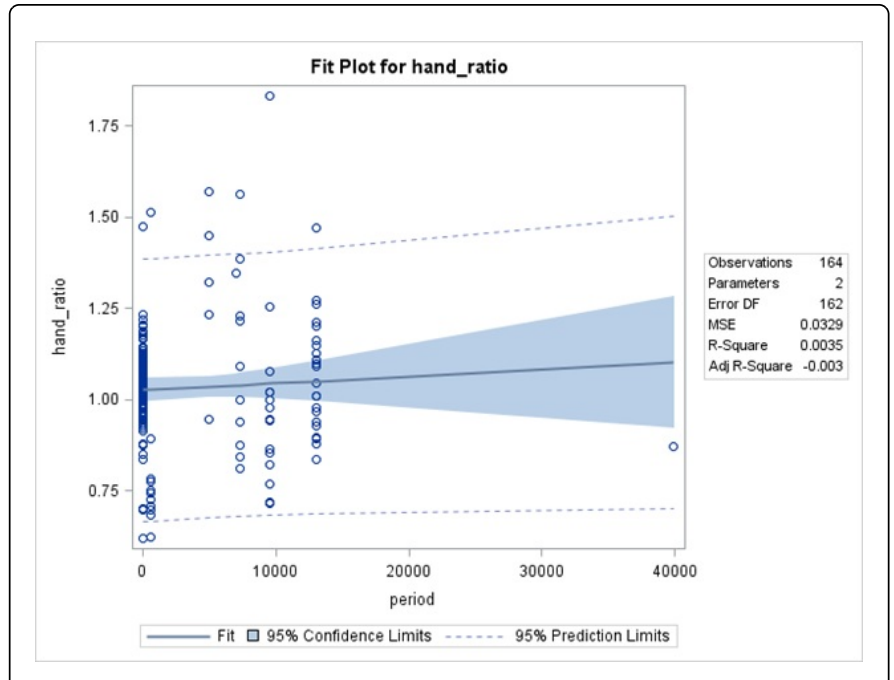

Figure 1: Relationship between hand ratios and period.

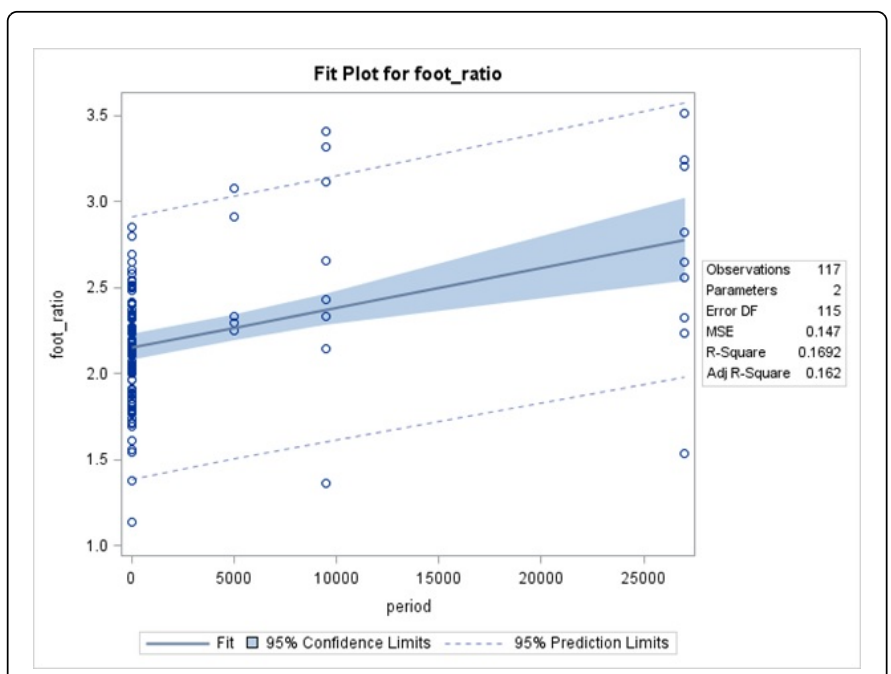

Figure 2: Relationship between foot ratios and period.

There was a significant difference between present and distant past in the slope $(\mathrm{P}<0.0001)$.

\section{Discussion}

Symbolic behaviors are dependent on the hands in humans whereas the foot is an instrument primarily for locomotion [4]. The function of hands in humans is uniquely adapted to the use of tools since Paleolithic times and appears not to have been a limiting factor in the evolution of gestures nor in symbolic behavior [5].

In contemporary artists, localized brain damage that occurs after stroke or trauma is not associated with impairment of artistic skills provided that the necessary motor functions remain preserved. This is attributed to extensive connectivity between the right and left hemispheres because artistic endeavors remain intact irrespective of damage to dominant or non-dominant hemispheres [2]. 
Compared to body mass the phalanges of the foot are very short. This is consistent with the evolutionary commitment to bipedal walking in humans. However, longer toes are advantageous in long distance running and could have been selected for when compared to the toe length in modern humans [6]. We show that the hand ratios compared over millennia remained essentially unchanged, whereas the foot ratios became significantly smaller in modern humans. Ratios are dimensionless numbers, therefore the foot ratios could have changed by either lengthening the toes or by broadening the foot. The likelihood of the foot broadening in increasingly tight modern footwear is therefore not a plausible explanation of the decrease in the foot ratios over time-the more likely explanation is a lengthening of the toes over millennia. This interpretation is also consistent with the shift from bipedal walking to modern mechanically assisted locomotion.

Anatomically modern humans (AMH) arrived in Europe $\sim 45,000$ years ago and rapidly spread, overlapping with Neanderthals for 2,600 5,400 years (at $95.4 \%$ probability) [7]. This had important implications for models seeking to explain the cultural, technological and biological elements involved in the replacement of Neanderthals with AMH. A mosaic of populations in Europe during the Middle to Upper Paleolithic transition suggests that there was ample time for the transmission of cultural and symbolic behaviors, as well as possible genetic exchanges in the two groups [8].

Humans started wearing shoes about 40,000 years ago. Because this changed the way humans walk and distribute weight while walking, from one foot to the other the human foot skeleton has changed over millennia [9]. People who don't wear shoes have wider feet and bigger gaps between their big toe and the other toes. And women who spend a lot of time on high heels have smaller calf muscles.

But, around 40,000 years ago, humans still had thick lower leg bones but their toes became shorter [10]. The results shown in Figure 2 (smaller foot ratios) are consistent with the skeletal changes in the lower limbs which first appeared $\sim 40,000$ years ago.

Human symbolic behavior was first noticed $\sim 40,000$ years ago also [2] in the appearance of hand and foot stencils in caves in various continents. But these activities and the manufacture of footwear would have required the development of brain connectivity related to the representation of hands and feet. It is not surprising, therefore, that both symbolic behaviors for the representation of hands and feet and footwear were approximately simultaneous.

Thus the advent of symbolic behavior such as cave stencils and the use of footwear seem to coincide with the advent of brain connectivity necessary for such behavior [11].

Hand stencils in South African caves have previously been examined [12]. Statistical analyses could be used in forensic work and other related endeavors as suggested but such analyses [12] require well developed brain connectivity and symbolic behavior such as reported in the present work.
More recently symbolic behavior has been demonstrated in Neandertals predating the arrival of modern humans in Europe by $\sim 100,000$ years using U-Th (uranium-thorium) dating of carbonate crusts from caves in Spain [13]. Thus modern techniques allowed the demonstration of brain connectivity necessary for symbolic behavior in both humans and Neandertals.

The results presented here are therefore consistent with the timescales of major changes in human behavior and lifestyles in Europe and with the evolution of human symbolic behavior.

\section{Acknowledgements}

Raffaella Bianucci thanks the Wellcome Trust for support of her research activity at Warwick Medical School, University of Warwick (UK). Otto Appenzeller is supported by the New Mexico Health Enhancement and Marathon Clinics Research Foundation.

\section{References}

1. Almecija S, Jeroen B, Smaers W, Jungers L (2015) The evolution of human and ape hand proportions. Nature Communications, pp: 1-11.

2. Zaidel DW (2010) Art and brain: insights from neuropsychology, biology and evolution. J Anat 216: 177-183.

3. Ocklenburg S, Schmitz J, Moinfar Z, Moser D, Klose R, et al. (2017) Epigenetic regulation of lateralized fetal spinal gene expression underlies hemispheric asymmetries. eLife 6: e22784.

4. Marzke MW, Marzke RF (2000) An approach to analysing and interpreting the anatomical evidence. J Anat 197: 121-140.

5. Povinelli DJ, Davis DR (1994) Differences between chimpanzees (Pan troglodytes) and humans (Homo sapiens) in the resting state of the index finger: implications for pointing. J Comp Psychol 108: 134-139.

6. Rolian C, Lieberman DE, Hamill J, Scott JW, Werbel W (2009) Walking, running and the evolution of short toes in humans. J Exp Biol 212: 713-721.

7. Fu Q, Posth C, Hajdinjak M, Petr M, Mallick S, et al. (2016) The genetic history of Ice Age Europe. (at 95.4\%probability). Nature 534: 200-205.

8. Higham T, Douka K, Jacobi R (2014) The timing and spatiotemporal patterning of Neanderthal disappearance. Nature 512: 306-309.

9. Nix S, Smith M, Vicenzino B (2010) Prevalence of hallux valgus in the general population: a systematic review and meta-analysis. J Foot Ankle Res 3: 21.

10. Duarte C, Maurício J, Pettitt PB, Trinkaus E, Plicht H, et al. (1999) The early Upper Paleolithic human skeleton from the Abrigo do Lagar Velho (Portugal) and modern human emergence in Iberia. PNAS 96: 7604-7609.

11. Tattersall I, Schwartz JH (1999) Hominids and hybrids: The place of Neanderthals in human evolution. PNAS 96: 7117-7119.

12. Henneberg M, Mathers K (1994) Reconstruction of body height, age and sex from handprints. South Afr J Sci 90: 493-496.

13. Hoffman DL, Standish CD, García-Diez M, Pettitt PB, Milton JA, et al. (2018) U-Th dating of carbonate crusts reveals Neandertal origin of Iberian cave art. Science 359: 912-915. 